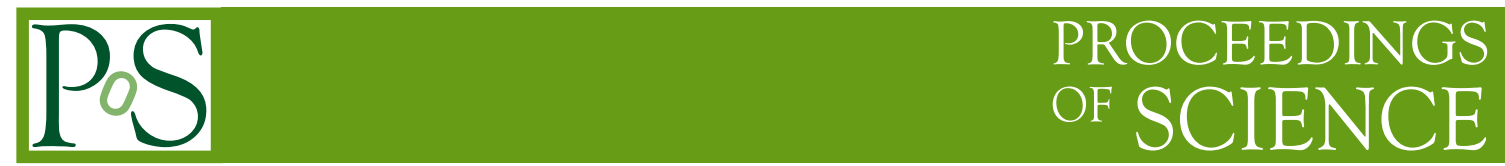

\title{
Current Status of the T2K experiment
}

\author{
Y. Oyama*† \\ KEK, Japan \\ E-mail: yuichi.oyama@kek.jp
}

The T2K long-baseline neutrino-oscillation experiment accumulated approximately $11.0 \times 10^{20}$ POT (protons on target) data until June 2015. The results of $v_{e}$ appearance as well as $v_{\mu}$ disappearance for $6.57 \times 10^{20}$ POT neutrino beam data are reported. A comparison with the results of reactor $v_{e}$ disappearance experiments provides possible hints toward negative $\delta_{\mathrm{CP}}$ phase with normal mass hierarchy. Preliminary results based on $4.04 \times 10^{20}$ POT anti-neutrino beam data are also presented.

18th International Conference From the Planck Scale to the Electroweak Scale 25-29 May 2015

Ioannina, Greece

* Speaker.

$\dagger$ on behalf of the $\mathrm{T} 2 \mathrm{~K}$ collaboration 


\section{Introduction}

In the present three-flavor picture of neutrinos, the flavor eigenstates is a mixture of mass eigenstates;

$$
\left(\begin{array}{c}
v_{e} \\
v_{\mu} \\
v_{\tau}
\end{array}\right)=\mathbf{U}_{\mathbf{M N S}}\left(\begin{array}{c}
v_{1} \\
v_{2} \\
v_{3}
\end{array}\right)
$$

The $3 \times 3$ unitary mixing matrix, $\mathbf{U}_{\mathbf{M N S}}$, is known as the Maki-Nakagawa-Sataka (MNS) mass matrix[1]. It has six independent parameters: two square-mass differences $\left(\Delta m_{21}^{2}\right.$ and $\left.\Delta m_{32}^{2}\right)$, three mixing angles $\left(\theta_{12}, \theta_{23}\right.$ and $\left.\theta_{13}\right)$ and one CP-violating phase $\left(\delta_{\mathrm{CP}}\right)$. The MNS matrix can be written as

$$
\mathbf{U}_{\mathbf{M N S}}=\left(\begin{array}{ccc}
1 & 0 & 0 \\
0 & \mathrm{c}_{23} & \mathrm{~s}_{23} \\
0 & -\mathrm{s}_{23} & \mathrm{c}_{23}
\end{array}\right)\left(\begin{array}{cccc}
\mathrm{c}_{13} & 0 & \mathrm{~s}_{13} e^{-i \delta_{\mathrm{CP}}} \\
0 & 1 & 0 \\
-\mathrm{s}_{13} e^{i \delta_{\mathrm{CP}}} & 0 & \mathrm{c}_{13}
\end{array}\right)\left(\begin{array}{ccc}
\mathrm{c}_{12} & \mathrm{~s}_{12} & 0 \\
-\mathrm{s}_{12} & \mathrm{c}_{12} & 0 \\
0 & 0 & 1
\end{array}\right)
$$

where $c_{\mathrm{ij}}=\cos \theta_{\mathrm{ij}}$ and $\mathrm{s}_{\mathrm{ij}}=\sin \theta_{\mathrm{ij}}$. The ranges of the oscillation parameters have been reported in many experiments, and are summarized by the particle data group[2] as listed in Table 1.

Among these measurements, the first indication of non-zero $\theta_{13}$ was claimed by the T2K (Tokai to Kamioka) experiment in 2011, based on $1.43 \times 10^{20}$ protons on target (POT)[3]. The result was confirmed by another long-baseline neutrino beam experiment[4], and three reactor experiments[5].

After the measurements of non-zero $\theta_{13}$, there are still open questions in neutrino oscillation physics. The two most important questions involve determining whether $\mathrm{CP}$ violation exists in the neutrino sector (the value of the CP phase) and defining the neutrino mass hierarchy, in other words, the sign of $\Delta m_{32}^{2}$.

$\mathrm{T} 2 \mathrm{~K}[6]$ is a long-baseline neutrino-oscillation experiment that began in 2009. A high-intensity neutrino beam from the J-PARC Main Ring is directed toward the Super-Kamiokande (SK) detec-

Table 1: Neutrino oscillation parameters reported in many experiments, and summarized by the particle data group[2]. NH indicates normal mass hierarchy, $m_{3}>m_{2}>m_{1}$, and IH indicates inverted mass hierarchy, $m_{2}>m_{1}>m_{3}$.

\begin{tabular}{lll}
\hline \hline $\sin ^{2} 2 \theta_{12}$ & $=0.846 \pm 0.021$ \\
$\Delta m_{21}^{2}$ & $=(7.53 \pm 0.18) \times 10^{-5} \mathrm{eV}^{2}$ \\
$\sin ^{2} 2 \theta_{23}$ & $=0.999_{-0.018}^{+0.001}(\mathrm{NH})$ \\
$\sin ^{2} 2 \theta_{23}$ & $=1.000_{-0.000}^{+0.000}(\mathrm{IH})$ \\
$\Delta m_{32}^{2}$ & $=(2.44 \pm 0.06) \times 10^{-3} \mathrm{eV}^{2}(\mathrm{NH})$ \\
$\Delta m_{23}^{2}$ & $=(2.52 \pm 0.07) \times 10^{-3} \mathrm{eV}^{2}(\mathrm{IH})$ \\
$\sin ^{2} 2 \theta_{13}$ & $=(0.093 \pm 0.008)$ \\
\hline
\end{tabular}


tor, $295 \mathrm{~km}$ away. This article provides updated results from the T2K experiment based on data accumulated until June 2015.

\section{T2K neutrino beam line and detectors}

A schematical view of the T2K neutrino beamline and detector components is shown in Fig.1. Details about them were previously reported in [7]. This article presents some of the important features.

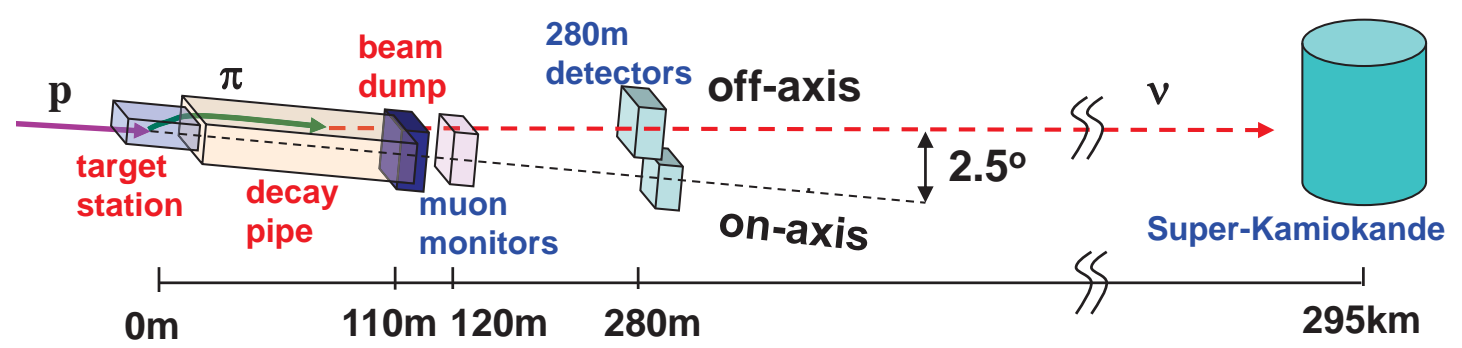

Figure 1: A schematical view of the T2K neutrino beamline and detectors. Beamline components are shown in red letters, and detectors are shown in blue letters.

\subsection{Neutrino beam line}

The proton beam for the T2K experiment is extracted from the J-PARC $30 \mathrm{GeV}$ Main Ring proton synchrotron. It is delivered to the carbon target in the target station. Pions and kaons are produced and subsequently bent in the direction of SK by a magnetic field produced by magnetic horns. Neutrinos are produced as decay products of pions and kaons. All particles except neutrinos and high energy $(>5 \mathrm{GeV}$ ) muons are absorbed by a beam dump located $110 \mathrm{~m}$ downstream of the target.

The most important feature of the T2K neutrino beam line is the off-axis beam, which was originally proposed in [8]. The conceptual idea regarding the off-axis angle in T2K was precisely reported in [9]. When the beamline construction was started, the off-axis angle was tunable[9] because the best off-axis angle was unknown. Based on information about the $\Delta m_{32}^{2}$ from other experiments[10,11], the off-axis angle was adjusted to $2.5^{\circ}$ in 2007 . The corresponding peak energy of the neutrino flux is approximately $600 \mathrm{MeV}$, and the oscillation study is most sensitive around $\Delta m_{32}^{2}=2.5 \times 10^{-3} \mathrm{eV}^{2}$.

\subsection{Muon monitors}

Two types of muon monitors are installed downstream of the beam dump. They are an Ionization Chamber and a Semiconductor Array. They can measure the intensity distribution of muons that have escaped from the beam dump. Because these muons are mainly produced along with neutrinos from the $\pi^{+}$decays, their beam center is in accordance with the beam center of neutrinos. The position of the beam center can be monitored on a bunch-by-bunch basis within a $3 \mathrm{~cm}$ resolution from the peak of the muon intensity distribution. This position resolution corresponds to a beam direction accuracy of $0.25 \mathrm{mrad}$. 


\subsection{Near detectors}

The near detectors were constructed in an underground experiment hall of $33.5 \mathrm{~m}$ depth and $17.5 \mathrm{~m}$ diameter at $280 \mathrm{~m}$ downstream from the target. Two detectors were installed; an on-axis detector (aimed in the direction of the neutrino beam center), and an off-axis detector (aimed in the direction of SK).

The on-axis detector, namely the INGRID detector, consists of $161 \mathrm{~m} \times 1 \mathrm{~m} \times 1 \mathrm{~m}$ cubic modules as shown in Fig.2(left). Each module is a "sandwich" of 11 scintillator layers and 10 iron layers. They are surrounded by four veto planes. The modules are arranged as follows: seven horizontally, seven vertically, and two off-diagonally. The neutrino beam center can be measured based on the horizontal/vertical distribution of the neutrino event rate.

The off-axis detector is named ND280. A schematical view of the ND280 detector is shown in Fig.2(right). The ND280 detector's purposes as a near detector are to measure the energy spectrum of muon neutrinos in SK's direction, and to measure the fractions of electron neutrinos.

All detector components except SMRD are placed inside a $0.2 \mathrm{~T}$ magnetic field produced by the recycled UA1 magnet from CERN. The P0D (Pi-zero Detector) is a subdetector component that is placed upstream inside the magnet. It is a "sandwich" of scintillator planes, lead plates, and a water target. It is customized for the measurement of neutral $\pi^{0}$ production. $\gamma$-rays from $\pi^{0} \rightarrow 2 \gamma$ are converted to electromagnetic showers by lead plates, and are detected by scintillators.

Downstream of the P0D, three TPCs (Time Projection Chambers) and two FGDs (Fine Grained Detectors) are placed. The TPCs can measure the momentum of muons from the curvature of the muon track in the magnetic field. The momentum resolution is greater than $10 \%$ at $1 \mathrm{GeV}$. FGDs consist of scintillator bars. They provide the target material for neutrino interactions, and are optimized for detecting the proton recoils. By combining TPCs and FGDs, the energy spectrum of $v_{\mu}$ can be precisely measured from CCQE (Charged Current Quasi-Elastic) neutrino interactions.

Other detector components, SMRD and ECAL, are installed in the area surrounding the POD and TPC/FGD. They are precisely reported in [7].
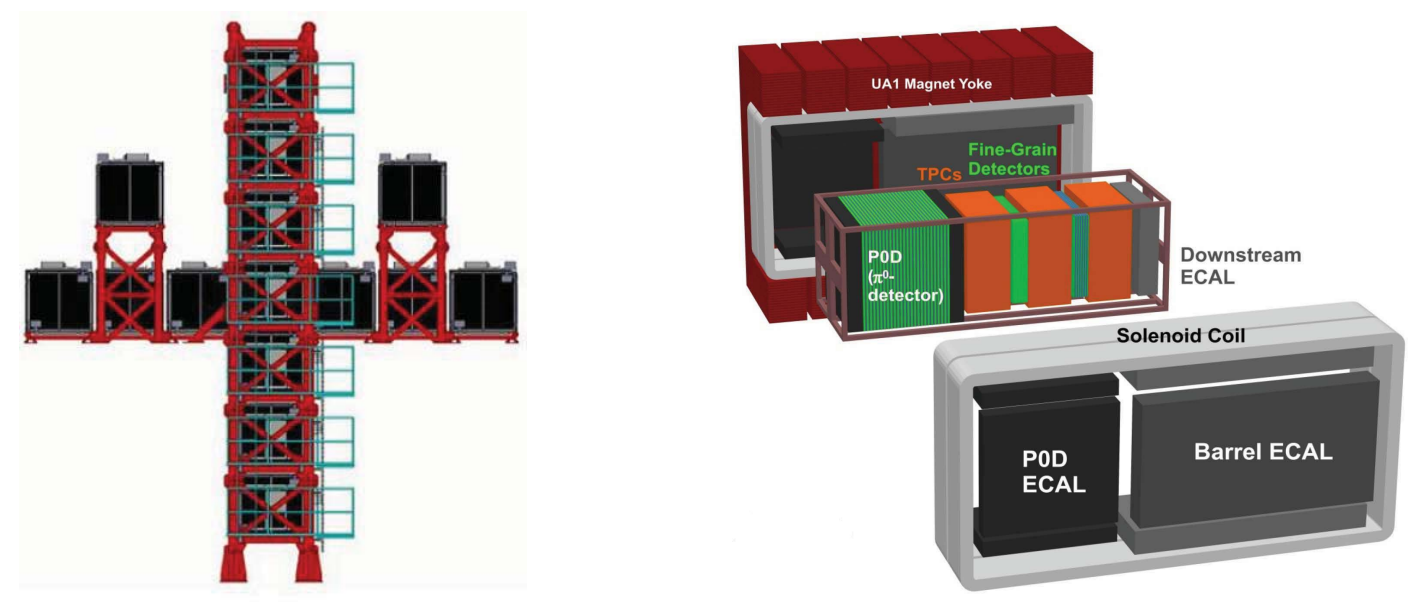

Figure 2: Schematic view of the INGRID on-axis detector (left), and the ND280 off-axis detector (right). 
The ND280 detector can be used as a near detector for the long-baseline neutrino-oscillation experiment, and as an independent detector for neutrino cross section measurements. Some results on the cross section measurements can be found in [12].

\subsection{Super-Kamiokande}

The far detector, Super-Kamiokande (SK), is a 50kton water Cherenkov detector[13] located $1000 \mathrm{~m}$ underground in the Kamioka mine, Japan. Its distance from J-PARC is $295 \mathrm{~km}$. In the inner detector (ID), 22.5kton of fiducial volume are viewed by 11,129 20-inch diameter PMTs. The outer detector (OD), which surrounds the ID, is also a water Cherenkov detector. It is used to veto events that enter or exit the ID. SK started its operation in April 1996. After a complete upgrade of its electronics systems in 2008, it was named SK-IV.

The most important characteristic of SK, as the far detector of the T2K experiment, is its ability to differentiate between muons and electrons. This particle identification directly implies identification between the parent $v_{\mu}$ (or $\bar{v}_{\mu}$ ) and $v_{e}$ (or $\bar{v}_{e}$ ). The principal of particle identification was reported in [14]. Muons passing through the detector are often unscattered due to their relatively large mass, and thereby produce clear ring patterns. Electrons, in contrast, scatter and produce electromagnetic showers, resulting in a diffuse ring edge. It was verified that probability of the $\mu / e$ misidentification is less than $1 \%[14]$.

\section{Status of the data-taking process}

The history of the proton beam delivery is shown in Fig.3. The physics data-taking began in January 2010. In early 2010 , one beam pulse had six bunches in $\sim 5 \mu$ s. The number of protons per pulse (ppp) was $\sim 2 \times 10^{13} \mathrm{ppp}$, and the beam pulse cycle time was 3.52 seconds.

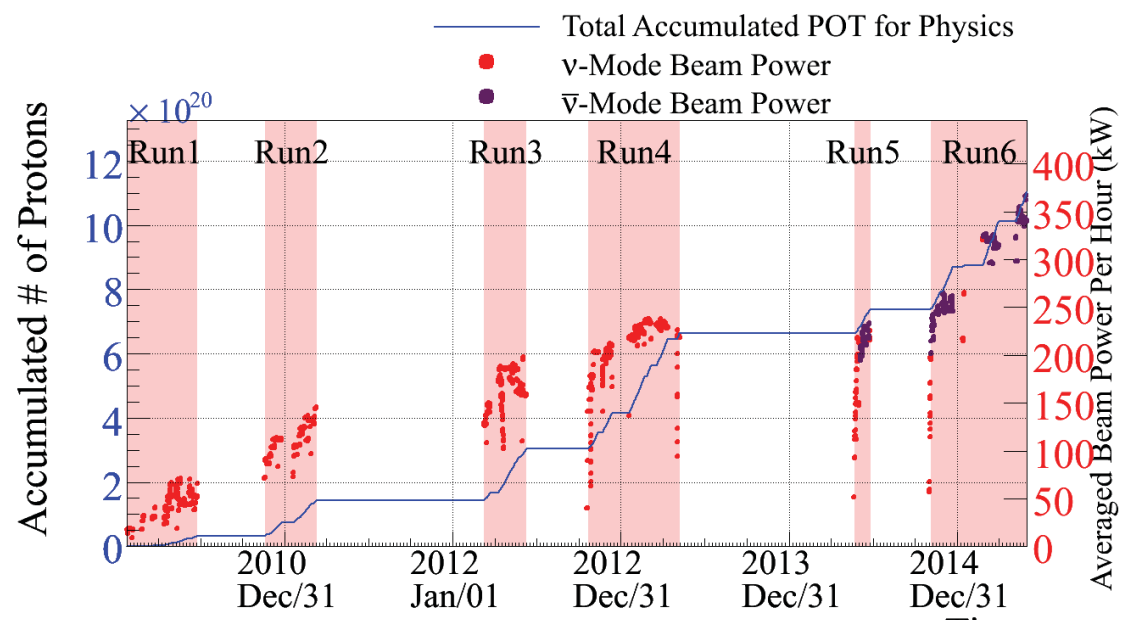

Time

Figure 3: History of primary proton beam intensity in the T2K experiment. Red (violet) dots show averaged beam power per hour in the neutrino (anti-neutrino) mode beam; the scale is given in the right vertical axis. The blue solid line shows the accumulated number of delivered protons from the beginning of the experiment. The scale is given in the left vertical axis. 
Many efforts were made by the J-PARC accelerator group to upgrade the beam power. In June 2015, the number of bunches in each pulse was 8 , and the number of protons per pulse was $\sim 1.8 \times 10^{14} \mathrm{ppp}$. The beam cycle time was upgraded to 2.48 seconds. The maximum beam power achieved through June 2015 was $371 \mathrm{~kW}$.

We accumulated $11.0 \times 10^{20}$ POT data until June 4,2015 . This is about $14 \%$ of our goal of $\sim 78 \times 10^{20}$ POT, which can be attained over five years of beam operation at a power of $750 \mathrm{~kW}$.

In June 2014, the direction of the magnetic horn current was reversed and an anti-neutrino beam run was started. The magnetic horns focus on the negative pions instead of the positive pions, and anti-neutrinos (as decay products of negative pions) can travel in a forward direction.

The stability of the beam direction is continuously monitored by the muon monitors and the INGRID detector as shown in Fig. 4. The beam direction is stable and well controlled within $\pm 1 \mathrm{mrad}$ accuracy. Note that if the beam direction is changed by $1 \mathrm{mrad}$, the neutrino flux at SK is changed by $2 \%$ at $E_{v}=0.5 \sim 0.7 \mathrm{GeV}$. Therefore, the accuracy of the beam direction is satisfactory. The stability of the neutrino event rate observed in the INGRID detector is also shown in Fig. 4.

In the next four sections, official results based on $6.57 \times 10^{20} \mathrm{POT}$ neutrino beam data accumulated until May 2013 are reported[15, 16, 17]. In sections 8 10, physics motivation and preliminary results based on $4.04 \times 10^{20} \mathrm{POT}$ anti-neutrino beam data recorded until June 2015 are reported[18].

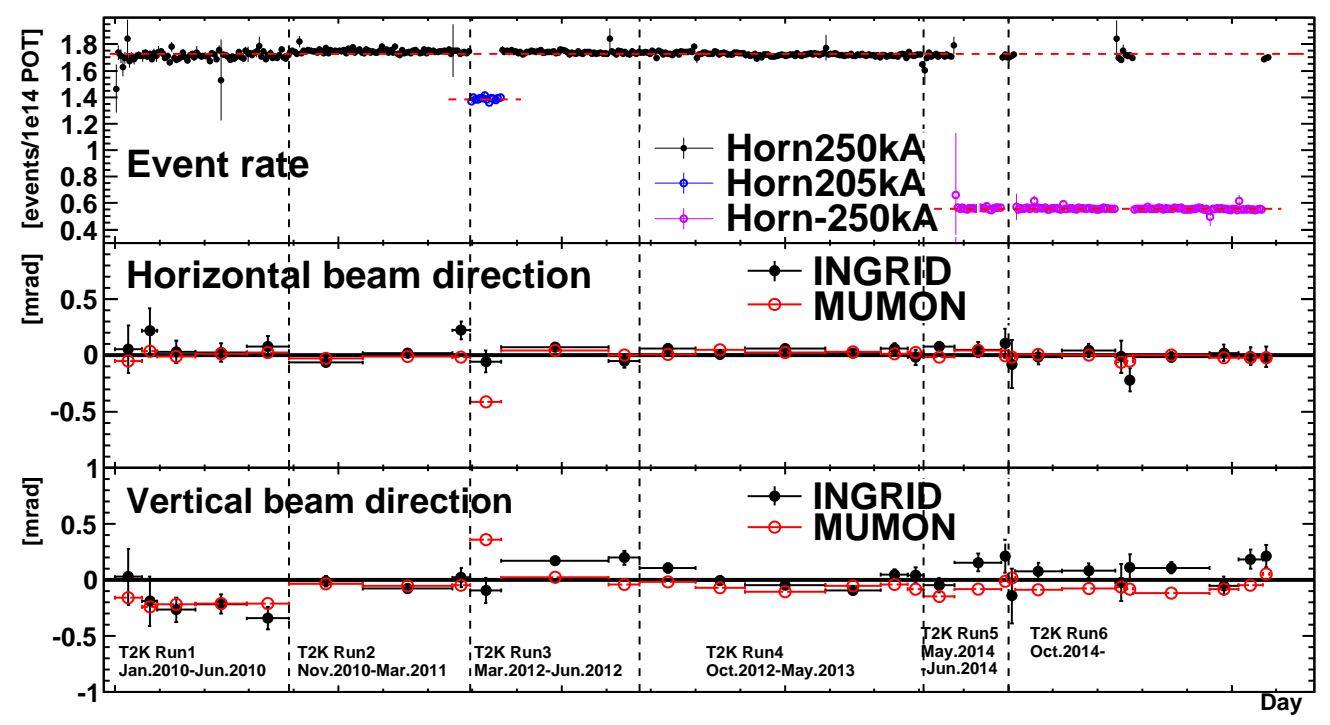

Figure 4: The top figure shows the event rate observed by the INGRID detector normalized by POT. The other two figures show muon beam directions measured by the INGRID detector and muon monitors. The beam direction is well controlled and is stable within $1 \mathrm{mrad}$. 


\section{Neutrino event selection}

Beam-related neutrino events are selected from SK data recorded during a $6.57 \times 10^{20} \mathrm{POT}$ beam period. The event selection process comprises two steps. The first step is the same for $v_{\mu}$ and $v_{e}$, and beam-related fully contained fiducial volume (FCFV) events are selected. Separate conditions are applied for $v_{\mu}$ and $v_{e}$ selection in the second steps. Details of the event selection are provided below.

Beam-related Fully Contained (FC) events are selected according to the following three conditions.

1) Total energy deposited in the ID is greater than $30 \mathrm{MeV}$

2) No outer detector activity

3) SK event time is within a range of -2 to $10 \mu \mathrm{sec}$ from the beam trigger time

The third condition examines correlations of the GPS time recorded in Tokai and in SK. A spatial reconstruction algorithm is applied to the FC events, and the following condition is applied.

4) The vertex position should be in the $22.5 \mathrm{kton}$ of fiducial volume

Note that the above analysis procedures except for step 3 are almost the same as the well-established atmospheric neutrino analysis[10].

After these conditions, 377 events are selected as FCFV (Fully Contained Fiducial Volume) events. The expected number of background events from non beam-related sources in accidental coincidence is estimated to be 0.0085 .

Table 2: Conditions of $v_{\mu}$ and $v_{e}$ event selection. Number of selected events for $6.57 \times 10^{20} \mathrm{POT} \mathrm{T} 2 \mathrm{~K}$ data and the expectation for no oscillation are also listed.

\begin{tabular}{|c|c|c|}
\hline & $v_{\mu}$ selection & $v_{e}$ selection \\
\hline \multirow[t]{9}{*}{ Conditions } & \multirow{4}{*}{\multicolumn{2}{|c|}{$\begin{array}{l}\text { 1) Total energy deposit in the ID }>30 \mathrm{MeV} \\
\text { 2)No outer detector activity } \\
\text { 3)SK event time agrees with the beam trigger time } \\
\text { 4) The vertex position should be in the } 22.5 \mathrm{kton} \text { fiducial volume }\end{array}$}} \\
\hline & & \\
\hline & & \\
\hline & & \\
\hline & $\mu$-1) $\mu$-like single ring & $e-1) e$-like single ring \\
\hline & $\mu-2) p_{\mu}>200 \mathrm{MeV} / \mathrm{c}$ & $e-2) p_{e}>100 \mathrm{MeV} / \mathrm{c}$ \\
\hline & $\mu-3) 0$ or 1 delayed-electron signal & $e-3) 0$ delayed-electron signal \\
\hline & & $e-4) E_{v}^{r e c}<1250 \mathrm{MeV}$ \\
\hline & & $e$-5)Tight $\pi^{0}$ rejection \\
\hline Number of selected events & 120 & 28 \\
\hline Number of expected events & $446 \pm 23$ (sys.) & $4.9 \pm 0.6$ (sys.) \\
\hline
\end{tabular}


A ring counting algorithm and the particle identification algorithm are applied to the 377 FCFV events. The events are categorized into single ring $\mu$-like events, single ring $e$-like events and multiring events.

In the $v_{\mu}$ event selection, the following three conditions are applied.

$$
\begin{aligned}
& \mu \text {-1) } \mu \text {-like single ring } \\
& \mu \text {-2) } p_{\mu}>200 \mathrm{MeV} / \mathrm{c} \\
& \mu \text {-3) } 0 \text { or } 1 \text { delayed-electron signal }
\end{aligned}
$$

In $\mu$-3), the delayed-electron signal is thought to be a Michel electron. After these conditions, 120 events remain as $v_{\mu}$ candidates.

In the $v_{e}$ event selection, five conditions are applied.

$$
\begin{aligned}
& e \text {-1) } e \text {-like single ring } \\
& e \text {-2) } p_{e}>100 \mathrm{MeV} / \mathrm{c} \\
& e-3) 0 \text { delayed-electron signal } \\
& e \text {-4) } E_{v}^{r e c}<1250 \mathrm{MeV} \\
& e \text {-5) Tight } \pi^{0} \text { rejection }
\end{aligned}
$$

By the condition $e-3$ ), events accompanied by Michel electrons are rejected. In the condition $e-4)$, the neutrino energy $E_{v}^{r e c}$ is calculated by assuming quasi-elastic kinematics. To suppress contributions by the intrinsic $v_{e}$ components arising primarily from kaon decays, the event is rejected if $E_{v}^{r e c}>1250 \mathrm{MeV}$. The condition $e-5$ ) is a special algorithm that suppresses contamination by misidentified $\pi^{0}$. The reconstruction of the second ring is forced, and the 2-ring invariant mass, $M_{i n v}$, is calculated. $M_{i n v}$ and the likelihood for $\pi^{0}$ are examined, and $\pi^{0}$-like events are rejected with tight criteria. Finally, 28 events remain after all selection criteria.

The conditions of the event selection and the results of the selection are summarized in Table 2.

\section{Expected events and their systematic errors}

The number of expected neutrino events in SK is carefully calculated by a Monte Carlo simulation of neutrino productions and their interactions.

From the interactions between primary proton beam and the carbon target, secondary pions and kaons are generated. Pions and kaons propagate in the secondary beamline. Neutrinos are produced as decay products of pions and kaons. All of these neutrino production processes are simulated by standard simulation programs, FLUKA[19, 20], GEANT3[21] and GCALOR[22]. To reduce the systematic uncertainties in pion and kaon productions, some of the T2K members joined the CERN NA61 experiment: "Study of hadron productions in hadron-nucleus and nucleusnucleus collisions in CERN SPS." Pion[23] and kaon[24] production data from a 30GeV proton beam and carbon target from NA61 are employed in the simulation. Systematic errors on neutrino fluxes in the $0.1 \mathrm{GeV} \sim 5 \mathrm{GeV}$ range at $\mathrm{ND} 280$ and SK are in the $10 \sim 15 \%$ range. However, fluxes 

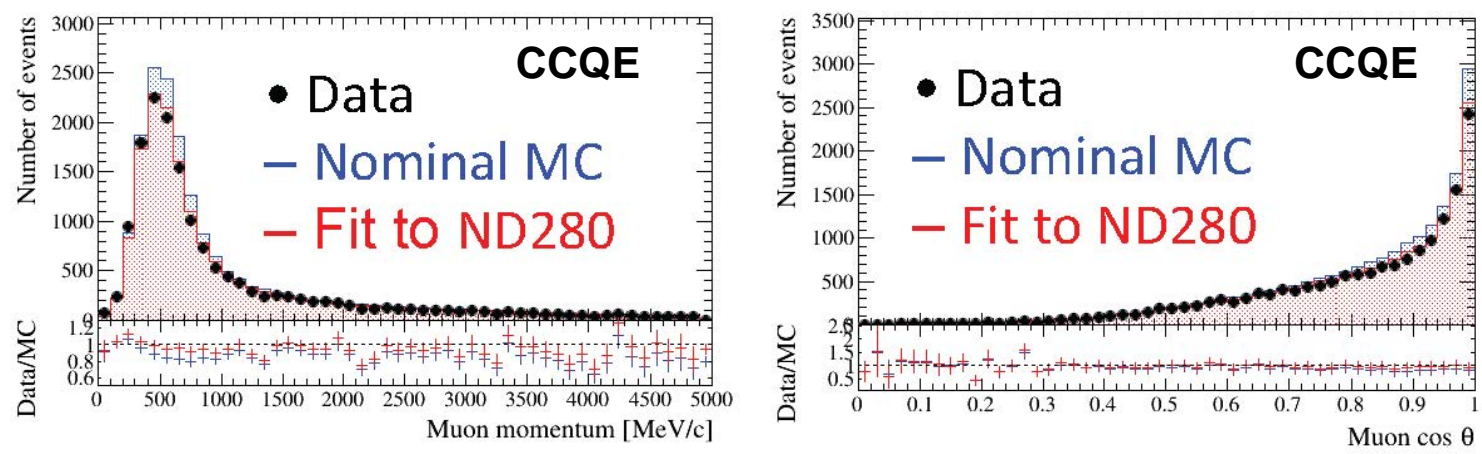

Figure 5: Momentum (left) and angle (right) distribution of CCQE events in ND280. After the adjustment of systematic uncertainties, agreements between the data and Monte Carlo simulation are excellent.

at the two detectors are highly correlated, and some of the systematic errors are common. An extrapolation of the ND280 analysis can reduce systematic errors in SK as discussed below.

The neutrino interactions are simulated with the NEUT[26] neutrino interaction generator. The GENIE[27] generator is also used to evaluate uncertainties caused by the simulation program. Model parameters in the neutrino interaction simulation, such as axial vector mass, are tuned by using external neutrino data. Results from MiniBooNe[25] are used as primary inputs. At present, nominal systematic errors for the neutrino cross section are larger than $\sim 10 \%$.

To reduce systematic errors, ND280 data are used. CCQE (Charged Current Quasi-Elastic) events, $\mathrm{CC} 1 \pi$ (Charged Current One Pion) events, and CCdis (Charged Current deep inelastic scattering) events are selected based on track topologies in ND280 FGD/TPC. $p_{\mu}$ and $\cos \theta_{\mu}$ distributions from the data and the simulation are carefully compared. Based on the comparison, all systematic errors related to cross sections and neutrino fluxes are adjusted. After the parameter adjustment $[15,16]$, the agreements are excellent as shown in Figure 5.

The adjusted parameters in the ND280 analysis can be also applied for SK. After the use of parameter adjustments, the systematic errors on expected neutrino events at SK are reduced from $23.5 \%$ to $7.7 \%$ for $v_{\mu}$ candidates, and from $26.8 \%$ to $6.8 \%$ for $v_{e}$ candidates. The extrapolation of ND280 analysis strongly reduces the systematic errors.

The event selection algorithm presented in section 4 was also applied to the Monte Carlo events. Numbers of expected neutrino event candidates for no oscillation are $446 \pm 23$ (sys.) for $v_{\mu}$ and $4.9 \pm 0.6$ (sys.) for $v_{e}$. The results are shown in Table 2.

\section{Results of muon neutrino disappearance}

As reported in the previous sections, 120 muon neutrino candidates are observed in $6.57 \times 10^{20}$ POT data, in which $446 \pm 23$ events are expected if no oscillation is assumed. The neutrino energy distribution for 120 events is also shown in Figure 6 (left). The disappearance of muon neutrino events as well as the distortion of the neutrino energy spectrum are obvious.

The best oscillation parameters are calculated by comparing expected events for given oscilla- 

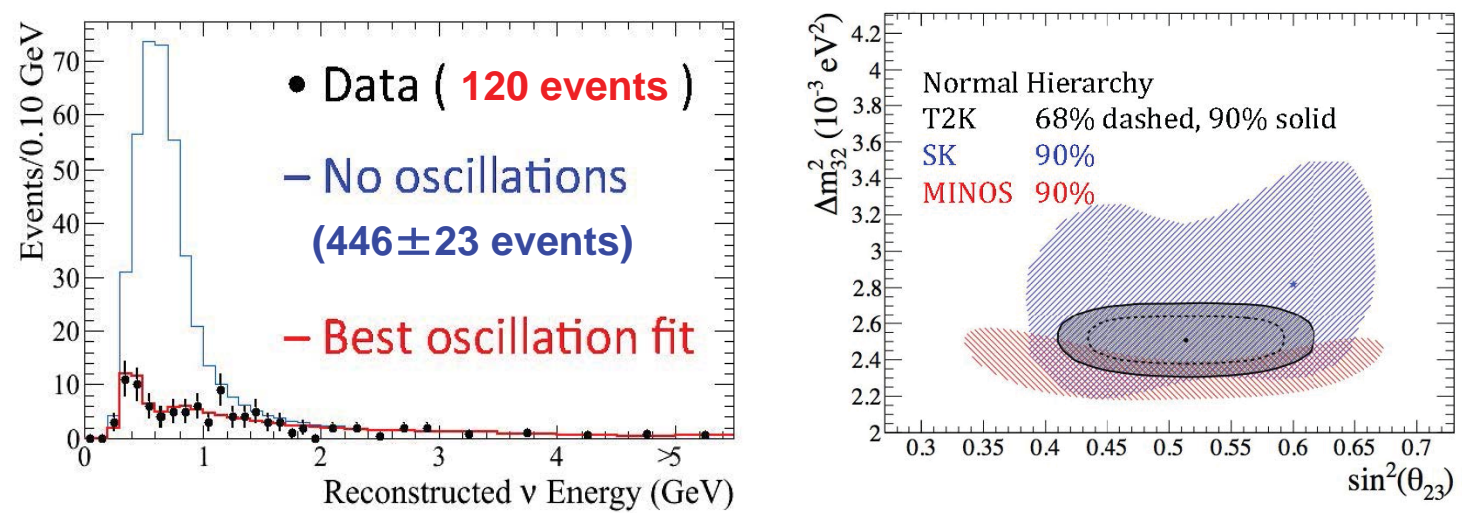

Figure 6: (left) Distribution of reconstructed neutrino energy for 120 muon neutrino candidates observed in $6.57 \times 10^{20}$ POT data. The expectations for no oscillation and for best-fit oscillation parameters are also shown. (right) Constraints on oscillation parameters $\Delta m_{32}^{2}$ and $\sin ^{2} \theta_{23}$ obtained from the disappearance of muon neutrino candidates. Normal mass hierarchy is assumed. Constraints from SK[28] and MINOS[29] are also shown.

tion parameters and the data. The best oscillation parameters were calculated as follows:

$$
\begin{aligned}
& \Delta m_{32}^{2}=(2.51 \pm 0.10) \times 10^{-3} \mathrm{eV}^{2} \\
& \sin ^{2} \theta_{23}=0.514_{-0.056}^{+0.055}
\end{aligned}
$$

for normal mass hierarchy, and

$$
\begin{aligned}
& \Delta m_{32}^{2}=(2.48 \pm 0.10) \times 10^{-3} \mathrm{eV}^{2} \\
& \sin ^{2} \theta_{23}=0.511 \pm 0.055
\end{aligned}
$$

for inverted mass hierarchy.

The constraint in the two dimensional $\sin ^{2} \theta_{23}-\Delta m_{32}^{2}$ plane for normal mass hierarchy is shown in Figure 6 (right), together with results from SK[28] and MINOS[29]. The T2K results are consistent with those of the two experiments, and provides the most stringent constraints for $\sin ^{2} \theta_{23}$.

\section{Results of electron neutrino appearance}

As presented in sections 4 and 5, 28 electron neutrino candidates are observed where $4.9 \pm 0.6$ events are expected for no oscillation. From a statistical calculation, the significance of the signal is 7.3 times the standard deviation. It can be concluded with certainty that this is discovery of the electron neutrino appearance. (Note that the T2K results based on $1.43 \times 10^{20} \mathrm{POT}$ data collected until 2011 was claimed as indication[3, 30], and the results based on the $3.01 \times 10^{20} \mathrm{POT}$ data collected until 2012 was claimed as evidence[31].)

Constraints on oscillation parameters are calculated by comparing the data and expectations. The allowed region in the $\sin ^{2} 2 \theta_{13}-\delta_{\mathrm{CP}}$ plane for normal mass hierarchy and inverted mass hierarchy are shown in Figure 7. The 68\% confidence level intervals for $\sin ^{2} \theta_{13}$ are 

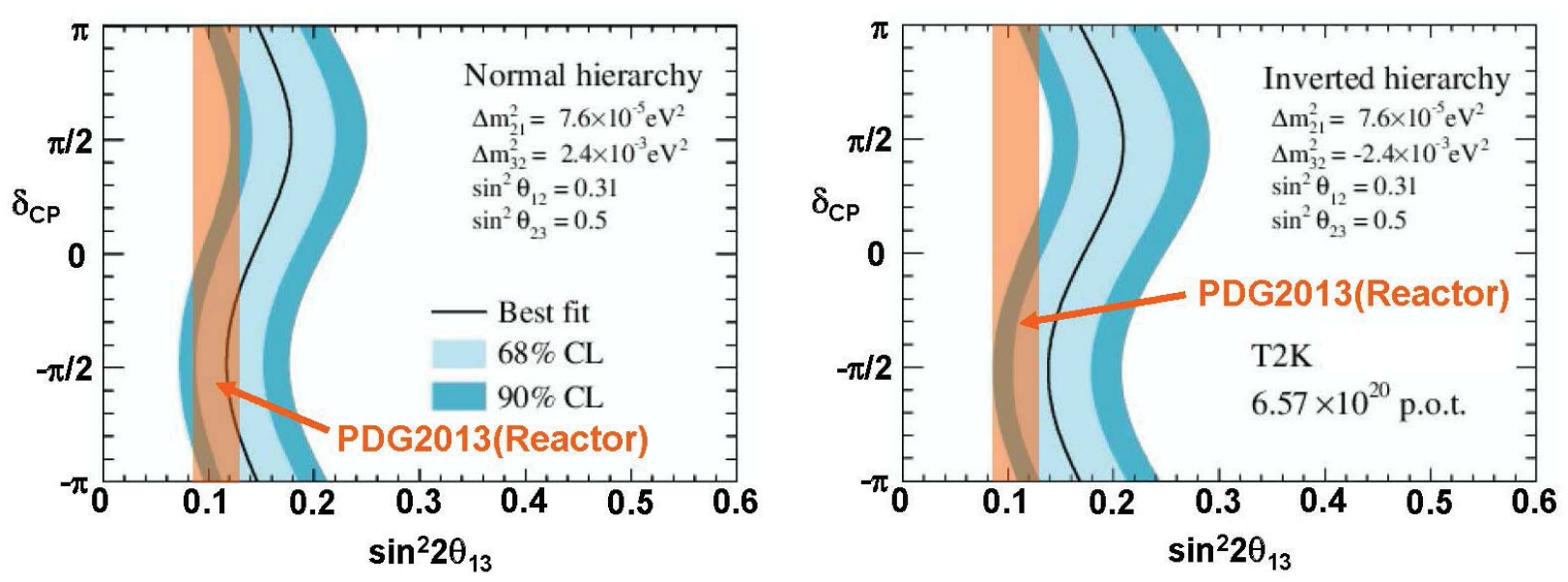

Figure 7: Constraints on oscillation parameters in $\sin ^{2} 2 \theta_{13}-\delta_{\mathrm{CP}}$ plane. Normal mass hierarchy (left) and inverted mass hierarchy (right) are assumed. Constraints on $\sin ^{2} 2 \theta_{13}$ from reactor experiments are also shown.

$$
\begin{array}{ll}
\sin ^{2} 2 \theta_{13}=0.140_{-0.032}^{+0.038} & \text { for normal mass hierarchy (NH) and } \\
\sin ^{2} 2 \theta_{13}=0.170_{-0.037}^{+0.045} & \text { for inverted mass hierarchy (IH) }
\end{array}
$$

if absence of a $\mathrm{CP}$ violation phase is assumed (i.e. $\delta_{\mathrm{CP}}=0$ ).

The constraints for $\sin ^{2} 2 \theta_{13}$ from reactor experiments[32] are also shown in Figure 7. The overlap between $\mathrm{T} 2 \mathrm{~K}$ and the reactor results indicates that negative $\delta_{\mathrm{CP}}$ with normal mass hierarchy is favored. More complicated and exhaustive statistical analysis methods[17] were employed, in which the muon neutrino disappearance and electron neutrino appearance are combined, and four parameters $\left(\delta_{\mathrm{CP}}, \theta_{13}, \theta_{23}\right.$ and $\left.\Delta m_{32}^{2}\right)$ are fitted simultaneously. From the analysis, the parameter ranges

$$
\begin{array}{ll}
0.146 \pi<\delta_{\mathrm{CP}}<0.825 \pi & \text { for normal mass hierarchy }(\mathrm{NH}) \quad \text { and } \\
-0.080 \pi<\delta_{\mathrm{CP}}<1.091 \pi & \text { for inverted mass hierarchy (IH) }
\end{array}
$$

are excluded with a $90 \%$ confidence level.

These results are considered to be the first hints toward $\delta_{\mathrm{CP}} \sim-\pi / 2$ and normal mass hierarchy. 

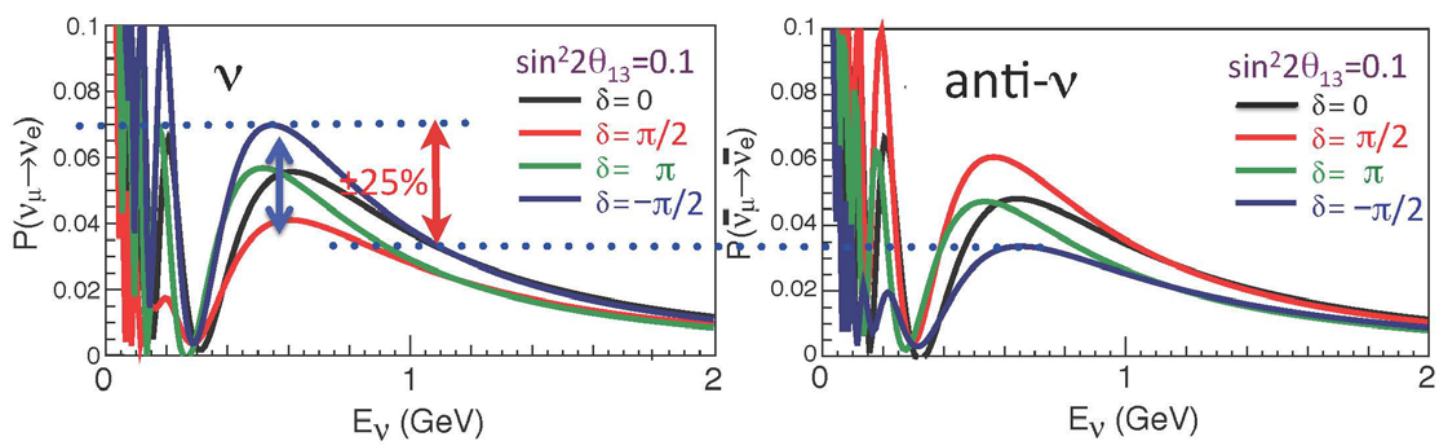

Figure 8: Oscillation probabilities for $v_{\mu} \rightarrow v_{e}$ (left) and for $\bar{v}_{\mu} \rightarrow \bar{v}_{e}$ (right) as functions of (anti-) neutrino energy. The neutrino travel distance is $295 \mathrm{~km}$. The anti-neutrino oscillation probability is larger (smaller) than the neutrino oscillation probability for positive (negative) $\delta_{\mathrm{CP}}$. The maximum difference is approximately $25 \%$.

\section{Motivation for anti-neutrino beam}

The probability for $\bar{v}_{\mu} \rightarrow \bar{v}_{e}$ oscillation can be written as follows;

$$
\begin{aligned}
P\left(\bar{v}_{\mu} \rightarrow \bar{v}_{e}\right) & \approx \sin ^{2} \theta_{23} \sin ^{2} 2 \theta_{13} \sin ^{2}\left(\frac{\Delta m_{32}^{2} L}{4 E_{v}}\right)\left(1+\frac{4 \sqrt{2} G_{F} n_{e} E}{\Delta m_{31}^{2}}\left(1-2 \sin ^{2} \theta_{13}\right)\right) \\
& +\sin 2 \theta_{12} \sin 2 \theta_{23} \sin 2 \theta_{13} \cos \theta_{13} \sin \delta_{\mathrm{CP}} \sin ^{2}\left(\frac{\Delta m_{32}^{2} L}{4 E_{v}}\right) \sin ^{2}\left(\frac{\Delta m_{21}^{2} L}{4 E_{v}}\right)
\end{aligned}
$$

It is slightly different from the probability for the $v_{\mu} \rightarrow v_{e}$ oscillation; the sign of $\delta_{\mathrm{CP}}$ term is not minus but plus for $\bar{v}_{\mu} \rightarrow \bar{v}_{e}$ oscillation. Because of this difference, the anti-neutrino oscillation probability is larger (smaller) than the neutrino oscillation probability for positive (negative) $\delta_{\mathrm{CP}}$ by up to $25 \%$ as shown in Figure 8 . Accordingly, comparison of oscillation probabilities between neutrinos and anti-neutrinos could determine the $\delta_{\mathrm{CP}}$ value.

The T2K group is planning to assign $50 \%$ of the beam time to the anti-neutrino beam run. Future sensitivity studies based on this condition are provided in [33]. The point of the plan is well explained by Figure 9. The shapes of allowed regions in $\sin ^{2} 2 \theta_{13}-\delta_{\mathrm{CP}}$ planes are different between neutrinos and anti-neutrinos. By combining two allowed regions, constraints for the $\delta_{\mathrm{CP}}$ range can be obtained in a single experiment without the use of reactor results.

As reported in section 3, the anti-neutrino beam started in June 2014, and $4.04 \times 10^{20} \mathrm{POT}$ data was accumulated until June 2015. In the following two sections, preliminary results for antineutrino beam data are presented[18]. Although the final goal is a measurement of $\bar{v}_{\mu} \rightarrow \bar{v}_{e}$ oscillation probability and a comparison with $v_{\mu} \rightarrow v_{e}$ probability, the first step is confirmation of $\bar{v}_{\mu}$ disappearance and $\bar{v}_{e}$ appearance. 

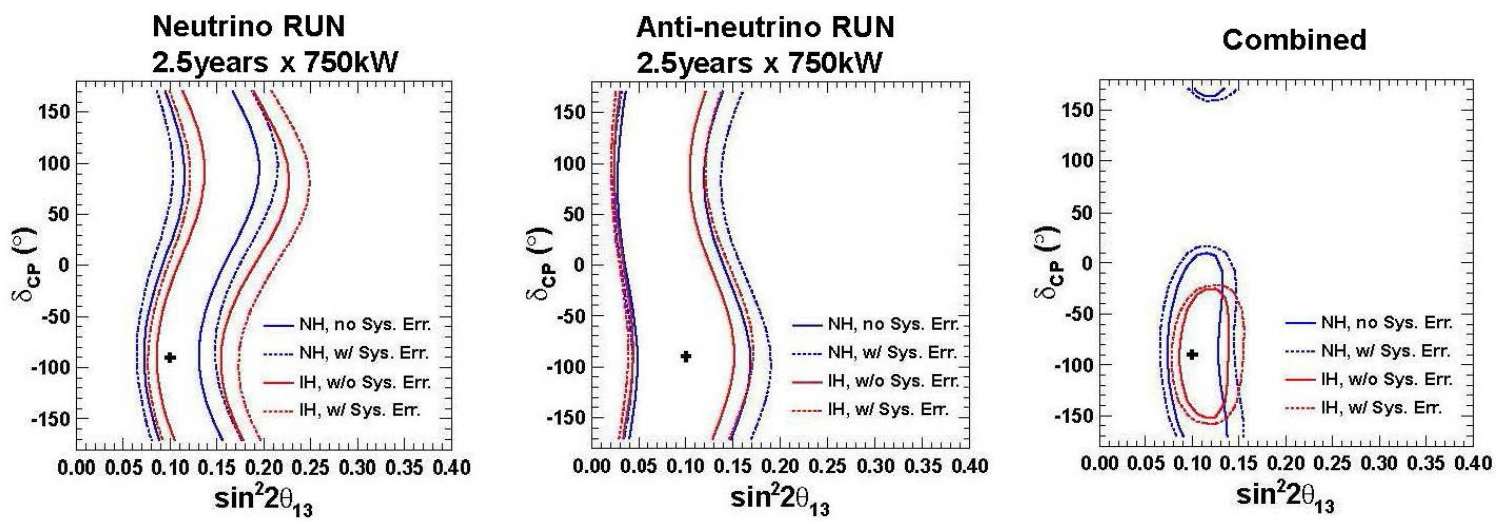

Figure 9: Expected $90 \%$ confidence level intervals in $\sin ^{2} 2 \theta_{13}-\delta_{\mathrm{CP}}$ planes. The true parameter values are assumed to be $\left(\sin ^{2} 2 \theta_{13}, \delta_{\mathrm{CP}}\right)=(0.10,-\pi / 2)$ with normal mass hierarchy, as indicated by "+". The left (center) plot is the expectation with 2.5 years of $750 \mathrm{~kW}$ neutrino (anti-neutrino) beam data. The right plot is the result of combining neutrino and anti-neutrino beam data. These plots were originally shown in Figure 3 of Ref [33].

\section{Preliminary results of $\bar{v}_{\mu}$ disappearance}

The disappearance of muon anti-neutrinos is examined using $4.04 \times 10^{20}$ POT anti-neutrino beam data. The analysis procedure that includes the event selection and calculation of the expectations by the Monte Carlo simulation are the same as those for neutrino analysis, and are not repeated here. In this case 103.6 events are expected for no oscillation and 34 events are observed.
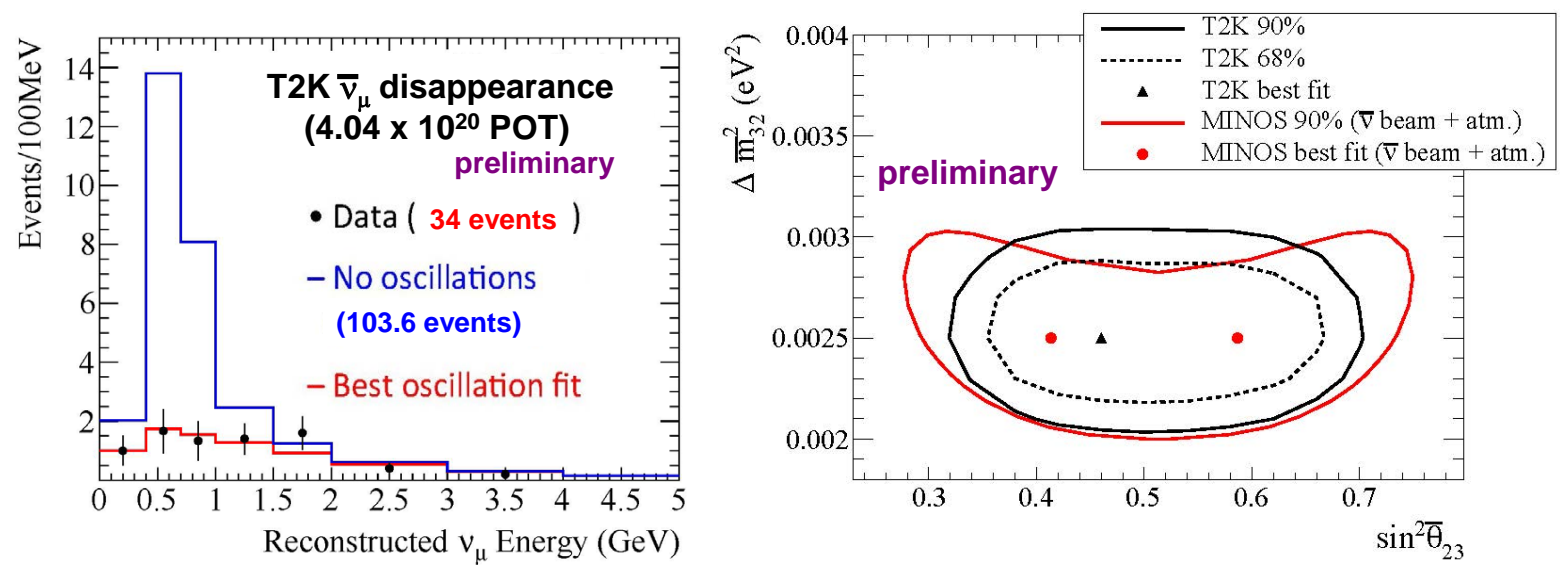

Figure 10: (left) Distribution of reconstructed neutrino energy for 34 single ring $\mu$-like events observed in $4.04 \times 10^{20}$ POT anti-neutrino beam data. The expectations for no oscillation and for best fit oscillation parameters are also shown. (right) Constraints on-oscillation parameters $\Delta \bar{m}_{32}^{2}$ and $\sin ^{2} \bar{\theta}_{23}$ obtained from $\bar{v}_{\mu}$ disappearance. Constraints from the MINOS[29] experiment are also shown. 
A reconstructed anti-neutrino energy spectrum is shown in Figure 10 (left). The reduction in event numbers as well as the distortion of the energy spectrum is obvious.

The best-fit oscillation parameters were calculated as follows:

$$
\begin{aligned}
\Delta \bar{m}_{32}^{2} & =\left(2.50_{-0.2}^{+0.3}\right) \times 10^{-3} \mathrm{eV}^{2} \\
\sin ^{2} \bar{\theta}_{23} & =0.46_{-0.06}^{+0.14}
\end{aligned}
$$

for normal mass hierarchy. No difference in best-fit parameters can be found for inverted mass hierarchy. No significant differences can be found between this section's results and those from the disappearance of muon neutrinos reported in section 6 .

The constraints on oscillation parameters in $\sin ^{2} \bar{\theta}_{23}-\Delta \bar{m}_{32}^{2}$ plane are calculated. The results for normal mass hierarchy are shown in Figure 10 (right) with results from the MINOS[29] experiment. The T2K results are consistent with MINOS. Again, more stringent constraints for $\sin ^{2} \bar{\theta}_{23}$ are obtained by $\mathrm{T} 2 \mathrm{~K}$.

\section{Preliminary results of $\bar{v}_{e}$ appearance}

The selection process of $\bar{v}_{e}$ candidates in SK is exactly the same as that for neutrino beam data. After all selections, three events remain as possible candidates of the $\bar{v}_{e}$ appearance signal.

The absence of $\bar{v}_{\mu} \rightarrow \bar{v}_{e}$ oscillation is assumed, and the expected numbers of background events are calculated. The background events include $v_{e}$ appearance from $v_{\mu} \rightarrow v_{e}$ oscillation, misidentified $v_{\mu}\left(\right.$ or $\left.\bar{v}_{\mu}\right)$, and original $v_{e}\left(\right.$ or $\left.\bar{v}_{e}\right)$ from the decay of muons in the T2K beam line. In these calculations, the existence of $v_{\mu} \rightarrow v_{e}$ oscillation with oscillation parameters by PDG2014[2] is assumed, although this assumption contradicts the absence of $\bar{v}_{\mu} \rightarrow \bar{v}_{e}$ oscillation within the MNS framework. The number of background events varies from 1.51 to 1.77 , depending on mass hierarchy and $\delta_{\mathrm{CP}}$. Obviously, the observation of three candidates is not significant evidence of $\bar{v}_{e}$ appearance.

The best-fit oscillation parameters obtained from neutrino beam analysis are assumed, and the number of expected $\bar{v}_{e}$ candidates are calculated for some $\delta_{\mathrm{CP}}$ with normal/inverted mass hierarchy. The results are 3.73 5.45 as summarized in Table 3. All of these numbers are not excluded by the $\mathrm{T} 2 \mathrm{~K}$ observation of the three events. Because of inadequate statistics, present T2K data cannot be used to examine any assumptions related to the oscillation parameters.

\section{Summary}

The T2K long-baseline neutrino-oscillation experiment accumulated $6.57 \times 10^{20}$ POT neutrino beam data and $4.04 \times 10^{20}$ POT anti-neutrino beam data until June 2015 .

In the $v_{\mu}$ disappearance analysis, 120 muon neutrino candidates are observed where the expectation for no oscillation is $446 \pm 23$. The disappearance of $v_{\mu}$ candidate events and the distortion of the neutrino energy spectrum are found. The best oscillation parameters were

$$
\begin{aligned}
& \Delta m_{32}^{2}=(2.51 \pm 0.10) \times 10^{-3} \mathrm{eV}^{2} \\
& \sin ^{2} \theta_{23}=0.514_{-0.056}^{+0.055}
\end{aligned}
$$


Table 3: Number of expected $\bar{v}_{e}$ candidates for $4.04 \times 10^{20} \mathrm{POT}$ T2K anti-neutrino beam. The results from T2K data are also listed.

\begin{tabular}{l|cc}
\hline \hline & $\delta_{\mathrm{CP}}$ & Number of $\bar{v}_{e}$ candidates \\
\hline Normal mass hierarchy & $-\pi / 2$ & 3.73 \\
& 0 & 4.32 \\
& $\pi / 2$ & 4.85 \\
\hline Inverted mass hierarchy & $-\pi / 2$ & 4.18 \\
& 0 & 4.85 \\
& $\pi / 2$ & 5.45 \\
\hline Data & & 3 \\
\hline \hline
\end{tabular}

for normal mass hierarchy. Constraints in $\sin ^{2} \theta_{23}-\Delta m_{32}^{2}$ plane are consistent with SK and MINOS. The most stringent constraints are obtained for $\sin ^{2} \theta_{23}$ by T2K.

In the $v_{e}$ appearance analysis, 28 electron neutrino candidates are observed where $4.9 \pm 0.6$ events are expected if no oscillation is assumed. The significance of the signal is 7.3 times the standard deviation, and it is certainly discovery of $v_{e}$ appearance. Results from reactors are combined, and the parameter range

$$
\begin{array}{ll}
0.146 \pi<\delta_{\mathrm{CP}}<0.825 \pi & \text { for normal mass hierarchy (NH) and } \\
-0.080 \pi<\delta_{\mathrm{CP}}<1.091 \pi & \text { for inverted mass hierarchy (IH) }
\end{array}
$$

are excluded with a $90 \%$ confidence level. These results are considered to be the first hints toward $\delta_{\mathrm{CP}} \sim-\pi / 2$ and normal mass hierarchy.

In the $\bar{v}_{\mu}$ disappearance analysis, $34 \bar{v}_{\mu}$ candidates are found where 103.6 events are expected for no oscillation. Distortion of the energy spectrum is also found, and the disappearance of $\bar{v}_{\mu}$ is obvious. The constraints on oscillation parameters, $\Delta \bar{m}_{32}^{2}$ and $\sin ^{2} \bar{\theta}_{23}$, are consistent with the results from the MINOS experiment. Any differences from parameters for neutrinos, $\Delta m_{32}^{2}$ and $\sin ^{2} \theta_{23}$, are identified.

In the $\bar{v}_{e}$ appearance analysis, three $\bar{v}_{e}$ candidates are found, where the numbers of background events are $1.51 \sim 1.77$, depending on mass hierarchy and $\delta_{\mathrm{CP}}$. Expected $\bar{v}_{e}$ candidates are calculated for some $\delta_{\mathrm{CP}}$ with normal/inverted mass hierarchy, and are 3.73 5.45. Present $\bar{v}_{e}$ appearance analysis cannot provide any constraints for the oscillation parameters, because of inadequate statistics. 


\section{References}

[1] Z.Maki, M.Nakagawa and S.Sakata, Prog.Theor.Phys. 28, 870 (1962).

[2] A.K.Olive et al. (Particle data group), Chinese Physics C 38, 090001(2014).

[3] K.Abe et al. (T2K Collaboration), Phys.Rev.Lett. 107, 041801 (2014).

[4] P.Adamson et al. (MINOS Collaboration), Phys.Rev.Lett. 107,181802 (2011).

[5] F.P.An et al. (Daya Bay Collaboration), Phys.Rev.Lett. 108, 171803 (2012); F.P.An et al. (Daya Bay Collaboration), Phys.Rev.Lett. 112, 061801 (2014);

J.K.Ahn et al. (RENO collaboration), Phys.Rev.Lett. 108, 191802 (2012);

Y. Abe et al. (Double Chooz Collaboration), Phys.Rev.Lett. 108, 131801 (2012).

[6] Y.Itow et al., hep-ex/0106019 (2001).

[7] K.Abe et al. (T2K Collaboration), Nucl.Instrum.Meth.A in press, arXiv:1106.1238 (2011).

[8] D.Beavis et al., BNL AGS E-889 (1995).

[9] Y.Oyama, hep-ex/0512041 (2005).

[10] Y.Ashie et al., Phys.Rev.D 71,112005 (2005).

[11] D.G.Michael et al., Phys.Rev.Lett. 97,191801 (2006).

[12] K.Abe et al. (T2K Collaboration), Phys.Rev.D 87, 092003 (2013);

K.Abe et al. (T2K Collaboration), Phys.Rev.D 89, 092003 (2014);

K.Abe et al. (T2K Collaboration), Phys.Rev.Lett. 113, 241803 (2014);

K.Abe et al. (T2K Collaboration), Phys.Rev.D 91, 112002 (2015).

[13] S.Fukuda et al., Nucl.Instrum.Meth.A 501, 418 (2003).

[14] S.Kasuga et al., Phys.Lett.B 374,238 (1996).

[15] K.Abe et al. (T2K Collaboration), Phys.Rev.Lett. 112, 181801 (2014).

[16] K.Abe et al. (T2K Collaboration), Phys.Rev.Lett. 112, 061802 (2014).

[17] K.Abe et al. (T2K Collaboration), Phys.Rev.D 91, 072010 (2015).

[18] M.Ravonel (on behalf of the T2K Collaboration), Talk at "European Physical Society HEP 2015", Vienna, July 22-29, 2015.

[19] A.Ferrari, P.R.Sala, A.Fasso, and J.Ranft, CERN-2005-010 and SLAC-R-773 and INFN-TC-05-11 (2005).

[20] G.Battistoni et al., AIP Conf. Proc. 896,31 (2007).

[21] R.Brun, F.Carminati and S.Giani, CERN-W5013 (1994).

[22] C.Zeitnitz and T.A.Gabriel, In Proc. of International Conference on Calorimetry in High Energy Physics, Tallahasse, FL, USA, February 1993.

[23] N. Abgrall et al. (NA61/SHINE Collaboration), Phys.Rev. C 84, 034604 (2011).

[24] N. Abgrall et al. (NA61/SHINE Collaboration), Phys.Rev. C 85, 035210 (2012).

[25] A. A. Aguilar-Arevalo et al. (MiniBooNE Collaboration), Phys.Rev.D 81, 092005 (2010);

A. A. Aguilar-Arevalo et al. (MiniBooNE Collaboration), Phys.Rev.D 83, 052007 (2011). 
[26] Y.Hayato, Nucl.Phys.(Proc. Suppl.) B112,171 (2002).

[27] C.Andreopoulos et al., Nucl.Instrum.Meth. A 614,87 (2010).

[28] A. Himmel (Super-Kamiokande Collaboration), AIP Conf. Proc. 1604, 345 (2014).

[29] P. Adamson et al. (MINOS Collaboration), Phys.Rev.Lett. 112, 191801 (2014).

[30] Y.Oyama, arXiv:1108.4457 (2011).

[31] K.Abe et al. (T2K Collaboration), Phys.Rev. D88, 032002 (2013).

[32] J. Beringer et al. (Particle Data Group), Phys.Rev. D86, 010001 (2012);

J. Beringer et al. (Particle Data Group), Phys.Rev. D86, 010001 (2013).

[33] K.Abe et al. (T2K Collaboration), Prog. Theor. Exp. Phys. 043 C01 (2015). 